\section{Bilateral intracerebral hemorrhage following CPR}

\author{
KYUNG CHUL CHA - TUYET NGUYEN THI - HYUNG JIN SHIN • YONG \\ SUNG CHA • HYUN KIM • KANG HYUN LEE • SUNG OH HWANG
}

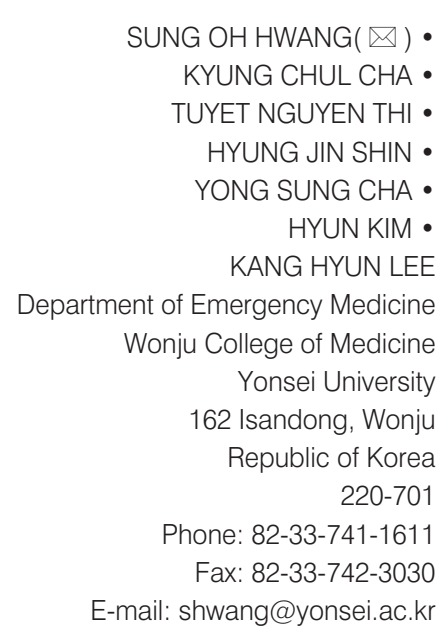

\begin{abstract}
Intracranial hemorrhage is an extremely rare complication following cardiopulmonary resuscitation (CPR). Only a few cases of subarachnoid hemorrhage during the post-resuscitation period have been reported in the literature.

A 55 year-old man, who was resuscitated following a cardiac arrest, sustained an intracranial hemorrhage during the post cardiac arrest period. His brain computerized tomography (CT), performed 4 hours after return of spontaneous circulation, showed no signs of intracranial hemorrhage. However, a brain CT performed 7 days after cardiac arrest revealed a massive intracerebral hemorrhage in both basal ganglia and thalami with an associated subarachnoid hemorrhage.

This paper reports the first case of intracerebral hemorrhages in bilateral basal ganglia and thalami that developed following cardiac arrest and resuscitation.
\end{abstract}

Key words: cardiopulmonary resuscitation (CPR), intracranial hemorrhage, basal ganglia hemorrhage, cardiac arrest

\section{Introduction}

Cardiopulmonary resuscitation (CPR) is a life-saving procedure for victims of cardiac arrest. However, CPR is frequently associated with complications. Complications of CPR are caused by external chest compressions or ventilatory support and are usually confined to the thorax and its adjacent structures. Frequent complications of CPR include fractures of the thoracic cage, tracheal injuries, and retropharyngeal bleeding caused by external chest compressions. (1) Injuries to extrathoracic organs are relatively rare complications of $\mathrm{CPR}$, and include liver injuries, gastric rupture, post-defibrillation rhabdomyolysis, spleen injuri- es, and air embolism. (2) Complications that occur in organs remote to the thorax are extraordinarily rare.

There have been a few reports of subarachnoid hemorrhage $(\mathrm{SAH})$ related to CPR in the literature. However, it is difficult to detect intracranial lesions that develop after CPR because most patients resuscitated from cardiac arrest remain unconscious in the early phase of the post-resuscitation period. This report describes a case of intracerebral hemorrhage in the bilateral basal ganglia and thalami that developed following cardiac arrest and resuscitation, and includes a review of the literature.

\section{Case report}

A 55 year-old man developed sudden dyspnea and lost consciousness. He had a 10 year history of diabetes mellitus. His collapse was witnessed by his wife, but bystander CPR was not performed. Emergency medical technicians arrived at the scene 25 minutes after his collapse and started to perform CPR, then transferred him to a local hospital. Initial electrocardiogram, at the first hospital, revealed asystole. Spontaneous circulation was restored after 11 minutes of advanced cardiovascular life support in the hospital. A large amount of food debris was aspirated via the endotracheal tube immediately after resuscitation. He was transferred to our hospital for management of post-cardiac arrest syndrome. On arrival, at our emergency department, his vital signs were as follows: blood pressure 69/43 $\mathrm{mmHg}$, heart rate 90 beats/min, and respiratory rate 35 breaths/minute. The patient was comatose, with a Glasgow Coma Scale score of 3 . His pupils were isocoric but were unresponsive to light. 


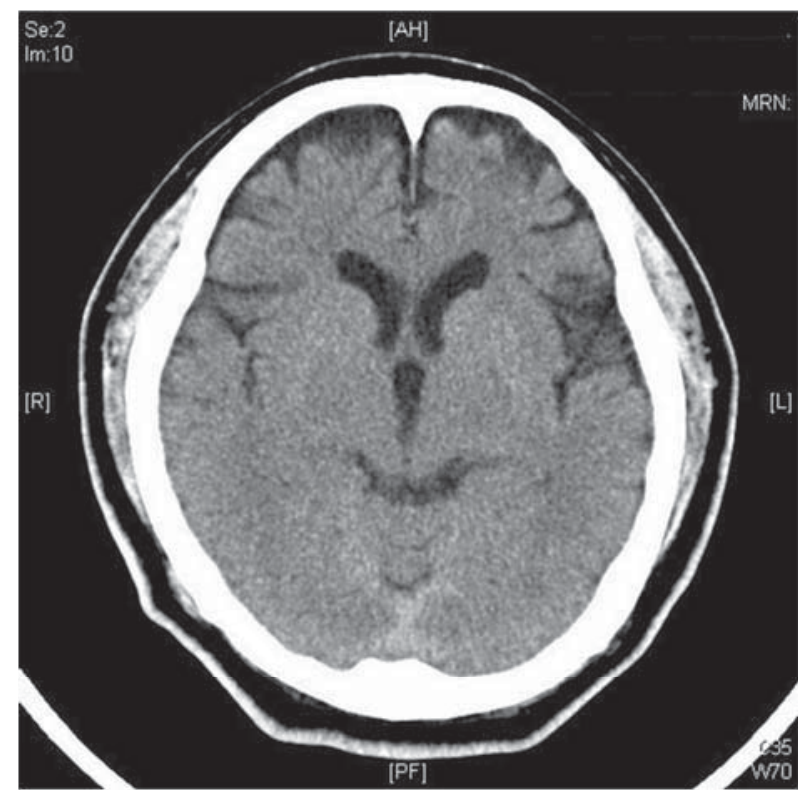

Figure 1. Findings of brain computerized tomography (CT) scan day 1 after admission.

The brain CT scan shows generalized hypodensity involving the entire cerebral hemisphere and loss of corticomedullary differentiation, suggesting global ischemia.

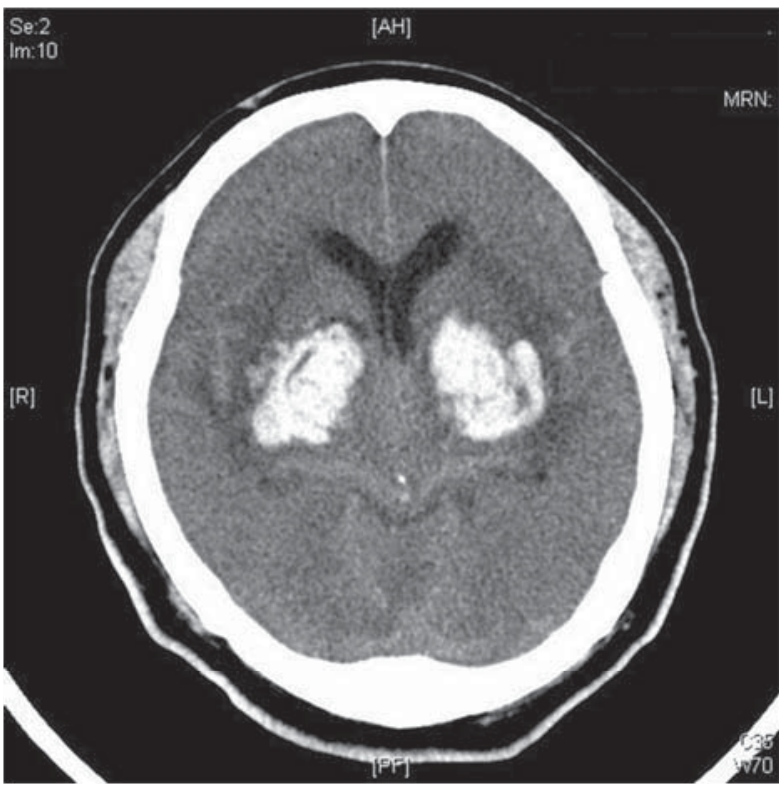

Figure 2. Findings of brain computerized tomography (CT) scan day 8 after admission.

The brain CT scan shows bilateral thalamic and basal ganglia hemorrhages with edema of the surrounding tissue and diffuse cerebral edema with effacement of all gray-white matter interfaces in both cerebral hemispheres. Subarachnoid hemorrhage is also noted.
Bony crepitus over the anterior thoracic wall and crackles over both lower lung fields were noted. No motor activity was observed. Systolic blood pressure was restored to $130 \mathrm{mmHg}$ with an infusion of a low dose of norepinephrine, and subsequently, the patient's hemodynamic status became stable. His complete blood count was normal on admission. The patient's platelet count began to decrease on the second day after arrest to $103,000 / \mathrm{mm}^{3}$ and returned to normal 7 days after arrest. The lowest platelet count was recorded on the fourth day, which was $58,000 / \mathrm{mm}^{3}$. On the day of admission, prothrombin time was 10.4 sec $(I N R=0.94)$, partial thromboplastin time was $36.2 \mathrm{sec}$ (control=32.3 sec), fibrinogen degradation products were $88.4 \mathrm{ug} / \mathrm{ml}$ (normal $<5 \mathrm{ug} / \mathrm{ml}$ ), D-dimer was $20,071 \mathrm{ng} / \mathrm{ml}$ (normal<280 ng/ $\mathrm{ml}$ ), activity of antithrombin-III was $73 \%$ (reference range: $77-123 \%$ activity), and fibrinogen was $366 \mathrm{mg} / \mathrm{dl}$ (200-400 mg/ dl). On the $4^{\text {th }}$ day of admission, prothrombin time was $15.1 \mathrm{sec}(\mathrm{INR}=1.36)$, partial thromboplastin time was 37.1 sec (control=32.3 sec), fibrinogen degradation products were $6.4 \mathrm{ug} / \mathrm{ml}$, D-dimer was $895 \mathrm{ng} / \mathrm{ml}$, antithrombin-III was $54 \%$ activity, and fibrinogen was > $700 \mathrm{mg} / \mathrm{dl}$.

Multiple patch-like infiltrates were detected in the patient's middle and lower lung fields. A brain computerized tomography (CT) performed 4 hours after return of spontaneous circulation (ROSC) showed no signs of intracranial hemorrhage (figure 1). The patient was transferred to the Intensive Care Unit (ICU) for post-cardiac arrest care including therapeutic hypothermia during the first 24 hours after ROSC. The patient remained comatose, but his pupils reacted to light from the second day of admission onward. Four days after admission, his level of consciousness remained unchanged. No brain stem reflexes were detected 6 days after cardiac arrest. A second brain CT scan was taken to evaluate possible causes of neurological change 7 days after cardiac arrest. Brain CT scans revealed massive intracerebral hemorrhages in both basal ganglia and thalami with an associated subarachnoid hemorrhage (figure 2). No specific management for intracranial hemorrhage was initiated considering the futility of treatment. The patient expired of respiratory failure from disseminated pneumonia 15 days after cardiac arrest.

\section{Discussion}

Intracranial hemorrhages $(\mathrm{ICH})$ are extremely rare complications or sequelae following cardiac arrest and CPR. Only one case report has described SAH following CPR. (3) It has been postulated that elevation of intracranial pressure secondary to chest compressions leads to hemorrhage in the subarachnoid space, especially in the presence of prior vascular malformations. (4) However, the exact mechanism of SAH following CPR remains unknown.

There have been no reports of intracranial parenchymal hemorrhage as a complication of CPR. To our knowledge, this is the first report of intracranial 
parenchymal hemorrhage that developed spontaneously during the postresuscitation period following cardiac arrest and CPR. In this patient, brain CT one day after cardiac arrest showed no evidence of $\mathrm{ICH}$, but the second brain CT, taken 7 days after resuscitation, showed a large amount of hemorrhage in both basal ganglia and thalami. This finding suggests that $\mathrm{ICH}$ was not the cause of sudden cardiac arrest but may have developed as a complication of cardiac arrest and resuscitation.

The exact mechanism of spontaneous $\mathrm{ICH}$ in this patient is unknown. One possibility is that $\mathrm{ICH}$ in this patient was a result of hemorrhagic transformation of brain infarction. Stasis of blood, increased levels of catecholamines, and endothelial damage during and after cardiac arrest activate coagulation mechanisms, and subsequent activation of endogenous fibrinolysis results in bleeding tendencies due to impairment of normal hemostasis. (5-7) Meanwhile, global cerebral ischemia due to diminution of blood flow over the entire brain during cardiac arrest and CPR causes hypoxic-ischemic brain injury. These conditions may lead to intracerebral bleeding due to hemorrhagic transformation of the ischemic area in prolonged CPR patients, similar to developing hemorrhage in patients with embolic stroke. (8-11) There have been several theories on the mechanism of hemorrhagic infarction, but in this particular patient, whose circulatory arrest time was prolonged over 30 minutes, the restitution of blood flow and subsequent development of coagulopathy may have resulted in hemorrhagic transformation of the ischemic infarction. Furthermore, it has been widely stated in the literature that the peak occurrence of hemorrhagic cerebral infarction is in the first week after stroke. $(11,12)$ Therefore, in this patient, the most appropriate mechanism may be the hemorrhagic transformation of brain infarction, which was due to global ischemia following prolonged CPR. Another possibility is that delayed hyperemia occurred during the post-cardiac arrest period. It has been demonstrated that the mean flow velocities of the middle cerebral artery begin to increase at 12 to 24 hours after CPR and peak 24 to 120 hours after CPR. (13) This delayed hyperemia can lead to intracranial hypertension, which results in delayed intracranial hemorrhage.

\section{Conclusion}

In conclusion, this report reveals that intracranial hemorrhage may develop following cardiac arrest and CPR. We recommend that during post CPR management, physicians should consider ordering brain CT scans in patients with deterioration of neurological status during the post-cardiac arrest period.

\section{REFERENCES}

1. Lederer W, Mair D, RabI W, Baubin M. Frequency of rib and sternum fractures associated with out-of-hospital cardiopulmonary resuscitation is underestimated by conventional chest X-ray. Resuscitation 2004 Feb;60(2):157-62.

2. Buschmann CT, Tsokos M. Frequent and rare complications of resuscitation attempts. Intensive Care Med 2009 Mar;35(3):397-404.

3. Lowenthal A, Maimon N, Waldman S, Almog Y. Sub-arachnoid hemorrhage following cardiopulmonary resuscitation. Resuscitation 2004 Nov;63(2):221-3.

4. Gueugniaud PY. Subarachnoid hemorrhage: a complication of CPR? Crit Care Med 1987 Mar;15(3):284-5.

5. Harukuni I BA. Mechanism of brain injury after global cerebral ischemia. Neurol Clin 2006;24:1-21.

6. Nolan JP, Neumar RW, Adrie C, Aibiki M, Berg RA, Böttiger BW, et al. Post-cardiac arrest syndrome: epidemiology, pathophysiology, treatment, and prognostication. A Scientific Statement from the International Liaison Committee on Resuscitation; the American Heart Association Emergency Cardiovascular Care Committee; the Council on Cardiovascular Surgery and Anesthesia; the Council on Cardiopulmonary, Perioperative, and Critical Care; the Council on Clinical Cardiology; the Council on Stroke. Resuscitation 2008;79:350-79.

7. Böttiger BW, Motsch J, Böhrer H, Böker T, Aulmann M, Nawroth PP, et al. Activation of blood coagulation after cardiac arrest is not balanced adequately by activation of endogenous fibrinolysis. Circulation 1995;92:2572-8.

8. Lodder J BK-KaJB. Cerebral hemorrhagic infarction at autopsy: cardiac embolic cause and the relationship to the cause of death. Stroke 1986;17:626-9.

9. Hornig CR WDaAA. Hemorrhagic cerebral infarction--a prospective study. Stroke 1986;17:179-85.

10. Okada Y, Yamaguchi T, Minematsu K, Miyashita T, Sawada T, Sadoshima S, et al. Hemorrhagic transformation in cerebral embolism. Stroke 1989;20:598-603

11. Ott BR, Zamani A, Kleefield J, Funkenstein HH. The clinical spectrum of hemorrhagic infarction. Stroke 1986;17:630-7.

12. Toni D, Fiorelli M, Bastianello S, Sacchetti ML, Sette G, Argentino C, et al. Hemorrhagic transformation of brain infarct: predictability in the first 5 hours from stroke onset and influence on clinical outcome. Neurology 1996;46:341-5.

13. lida K, Satoh H, Arita K, Nakahara T, Kurisu K, Ohtani M. Delayed hyperemia causing intracranial hypertension after cardiopulmonary resuscitation. Crit Care Med 1997 Jun;25(6):971-6. 